\title{
AGRICULTURAL FIELDS MONITORING WITH MULTI-TEMPORAL POLARIMETRIC SAR (MT-POLSAR) CHANGE DETECTION
}

\author{
Cristian Silva-Perez \\ University of Stirling, Scotland, United Kingdom \\ Armando Marino \\ University of Stirling, Scotland, United Kingdom vspace1em \\ Juan M. Lopez-Sanchez \\ University of Alicante, IUII, Alicante, Spain \\ Iain Cameron \\ Environment systems Ltd, Aberystwyth, United Kingdom
}

This work presents a novel methodology to extract and analyse multitemporal polarimetric SAR (PolSAR) information from a stack of co-registered images. The method is based on the analysis of PolSAR changes between every image with respect to the rest of images in the stack. The changes are organized in a matrix form to encode the polarimetric evolution of a target. The change matrix is then used to visually understand a target evolution and its SAR response based on the evolution of scattering mechanisms due to the target physical evolution. Additionally, we design and test an image classification algorithm in a supervised learning fashion by using typical change matrices as training data. The methodology is tested exploiting C-band quad-pol RADARSAT-2 data with special interest on agricultural fields such as rice in Seville, South-West of Spain and in the Indian Head in Canada as part of the Agrisar 2009 campaign. 


\section{Introduction}

To monitor land dynamics from Synthetic Aperture Radar (SAR), multi-temporal analysis is commonly used in order to characterize the sensor response to different stages of a target. This is particularly important for agricultural fields monitoring since an initial step is to understand the PolSAR evolution in time over a crop due to changes in the plant's morphology. Previously a methodology to study the evolution of the PolSAR measurements in time was introduced, with the aim of understanding the SAR response to the intensity and type of changes that occurred in a target between a pair of consecutive acquisitions [1-3].

The present work extends the same methodology since it not only determines changes in scattering mechanisms (SM's) of consecutive images but also the determines the pair-wise changes between all the images in the stack. The RGB results are organized as a matrix so that the ith row represents the change from the ith acquisition to all the other acquisitions. The change matrix allows to understand and visualise the interaction of the SAR signal with a target, i.e an agricultural field, throughout a season. The principle can be applied to any object on the ground and a change matrix can be built for each resolution cell in an image.

In this sense, the change matrix encodes the PolSAR multitemporal information of a target along a season. Using the encoded information provided by the matrix, we compare the similarity between a pixel change matrix and some model change matrices (classes) in a supervised learning fashion. Thus, we can label any pixel in the image of belonging to the class with the most similar change matrix. The change matrix is then used to understand the PolSAR evolution of a target and as the

core of a multi-temporal classification methodology to determine the land use/land cover. 


\section{Test site and Datasets}

The methodology is initially applied to a location near Seville, in the south of Spain as shown in figure 1. It includes several land types, particularly including rice fields where ground truth is known.

The satellite data used is composed of 15 C-band quad polarimetric Radarsat-2 Images divided in three series of 5 images, each series with a different incidence angle during the 2014 season, covering from May to September. To show how different the change matrices are for different crop types, the same methodology was applied to several crops including cereals such as barley, oats and wheat and other crop types such as canola, and field peas. The crops are located in the Indian head (Canada), where ground truth was gathered as part of the Agrisar 2009 campaign.

\section{Change detector}

The proposed methodology uses a polarimetric change detector as the core of its operation. It is based on the analysis of a pair of co-registered acquisitions by optimizing the pixel-wise difference of their PolSAR covariance matrices. The optimization process uses the diagonalization of this difference matrix as described in [2,3], to find the set of unitary vectors(eigenvectors) that cause the largest polarimetric change between the pair of images. The output provides information about the type and intensity of the SM's that were added and/or removed from one image to the other. This can also be seen as finding the SM's that increased and decreased between images.

The SM's are represented as RGB composites where each element of the eigenvector takes a colour and the contrast of the image is modulated by its associated eigenvalue.

\section{Multitemporal change matrix}

Since the information about the crop evolution is obtained from the analysis of pairs of images, it is possible to evaluate not only changes from consecutive images (as 


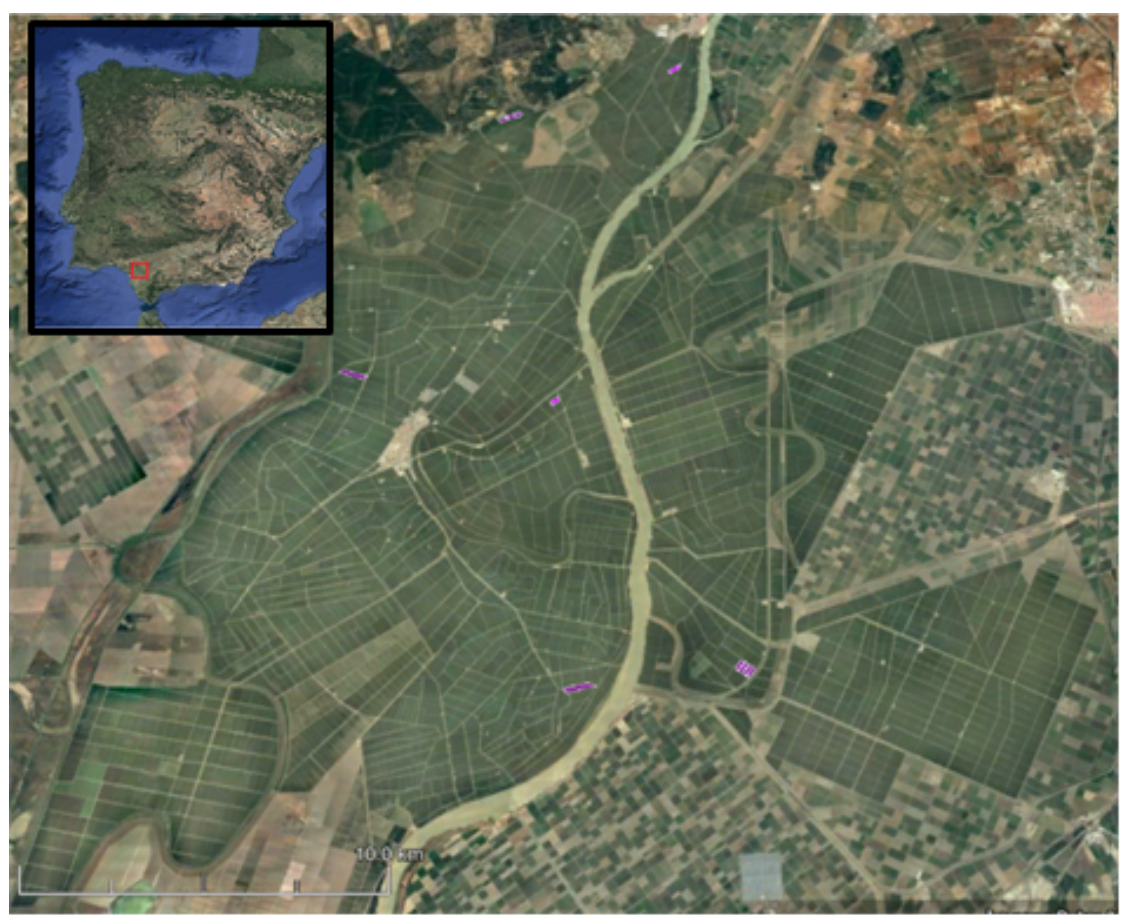

(a) Google earth

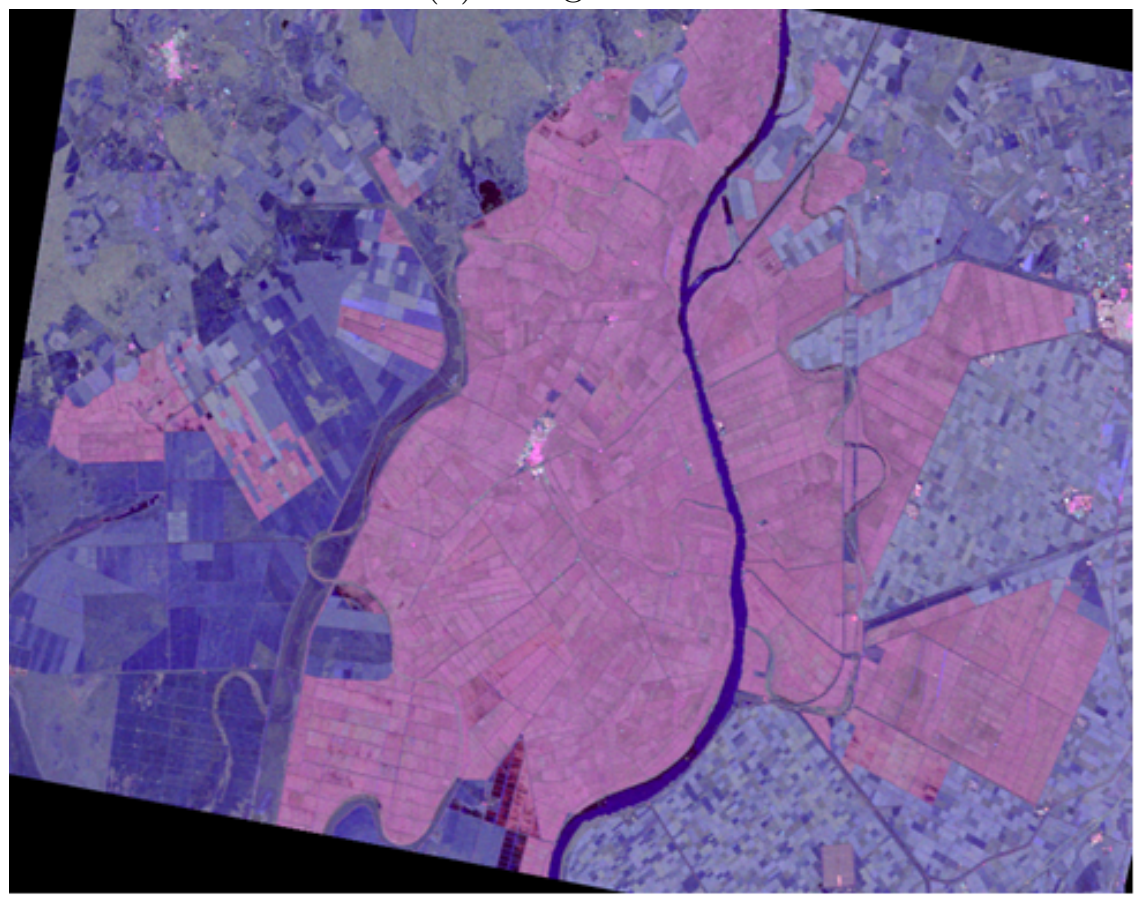

(b) Pauli RGB composite 2014/08/16

Figure 1. Test site in the South of Spain 
traditionally done) but is also possible to see the difference of an image with respect to all the other images in the stack. This process identifies the change of the scattering mechanisms throughout the season.

The information can be represented by a square matrix, where each element of the diagonal represents one of the acquisitions. The off-diagonal elements correspond to the evaluation of polarimetric changes between images. The upper triangular part represents the increase in scattering mechanisms between a given pair of images and the lower triangular part represents the corresponding decrease. It is worth noticing that the unitary vector that represents a scattering mechanism is shown as an RGB composite using its associated eigenvalue to modulate the contrast.

\section{Rice crop change matrices}

The result of this process is shown in figure 2 for the rice fields. The figures 2.a) to 2.c) represent the change matrix for three different parcels where ground truth is known.

A remarkable characteristic of the matrix is that its colours and intensity provide straight forward information of the scattering mechanisms during the season and they can be interpreted as a conventional RGB composite, that is, red for double bounce, green for volume and blue for surface scattering. This represents a valuable insight to understand a crop evolution and its SAR response without the need for ground truth but only based on the evolution of scattering mechanisms due to the physical processes related to crop growth.

It can be seen in figures 2(a) and 2(b) that these rice crop change matrices of two different parcels have minor differences corresponding to the normal differences in the parcel's evolution. However, the general patterns as well as the colours and intensity are preserved. The parcel of figure (c) on the other hand, presents an atypical

evolution of scattering mechanisms which coincides with the information from the ground truth (delayed plant growth). It is important to notice that because the matrix 


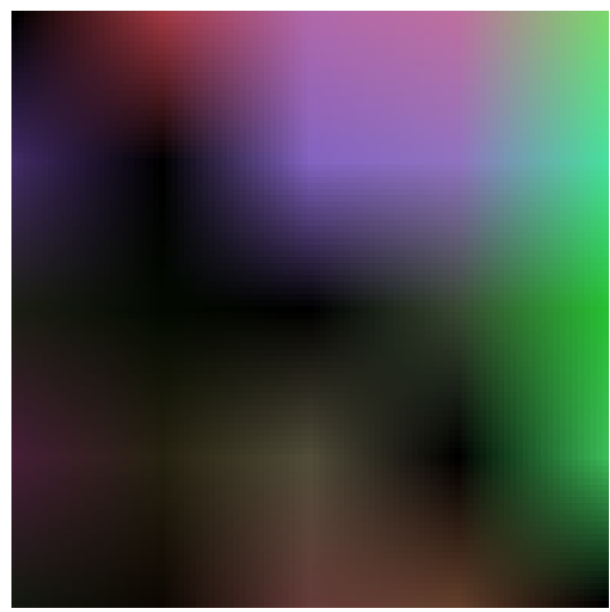

(a) Parcel E

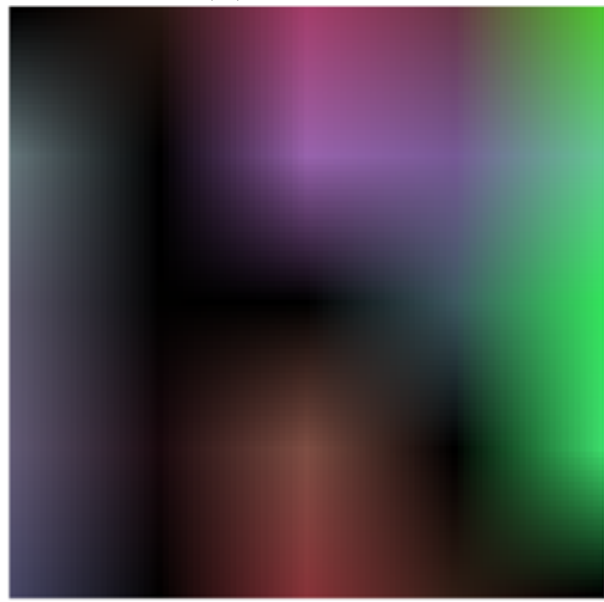

(b) Parcel F

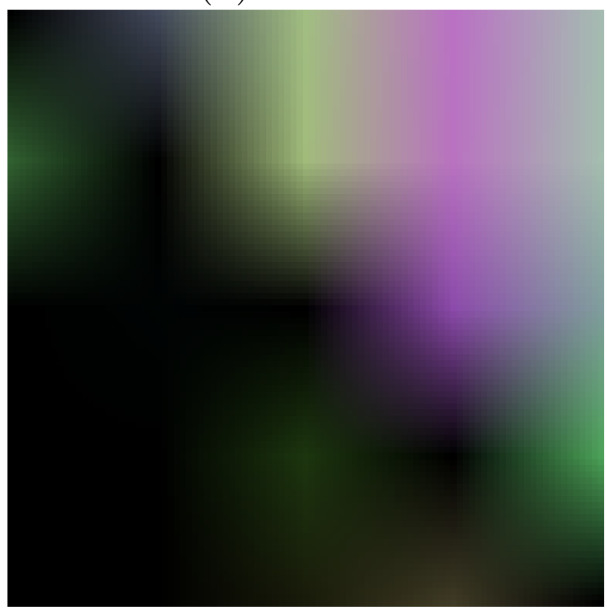

(c) Parcel B

Figure 2. RGB composite of the rice fields change matrices. RADARSAT-2 quadpol data season of 2014 
contains separately the increase and decrease in time of each scattering mechanism, the analysis of parcel evolution can be done for each mechanism separately. This results in being able to compare for instance if the double bounce in a parcel due to the plants emergence above the water level is delayed in time with respect to a reference parcel.

Furthermore, since each row and column of the matrix is associated with a day of the year, the change matrix immediately tells the scattering mechanism present at any given moment during the season (it can also be associated with a ground truth variable e.g. phenological stage at a particular day).

\section{Change matrix for different land types}

The same methodology was applied to other crops including cereals such as barley, oats and wheat and other crop types such as canola, and field peas. The crops are located in the Indian head (Canada), where ground truth was gathered as part of the Agrisar 2009 campaign. A stack of 10 Radarsat-2 Quad Pol images was analysed, and the resulting change matrices are shown in the figure 3

It is possible to see by visual inspection how different the resulting matrices are for each of these three crop-types and to that of the rice crop presented in figure 2 . These differences, described by the colours that represent the scattering mechanisms, the contrast of the image and the times when specific events occur, can be used as a feature for image classification, in this case crop type classification.

Also significant differences can be seen for the other land types as shown in figure 4, confirming the usefulness of the change matrix to encode the evolution of a target in the ground. 


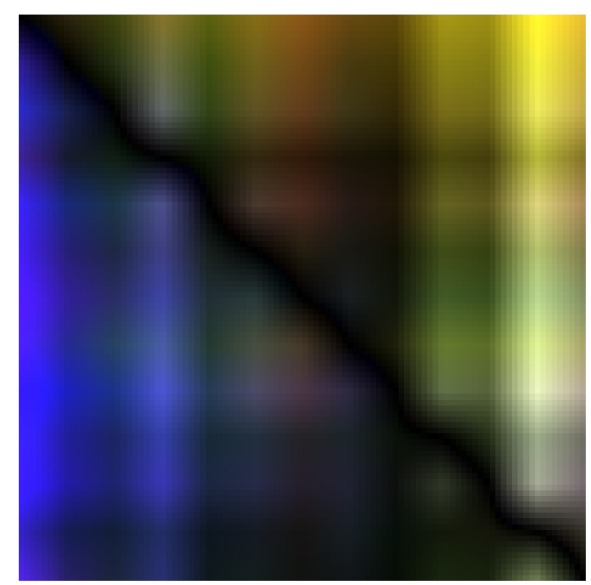

(a) Barley

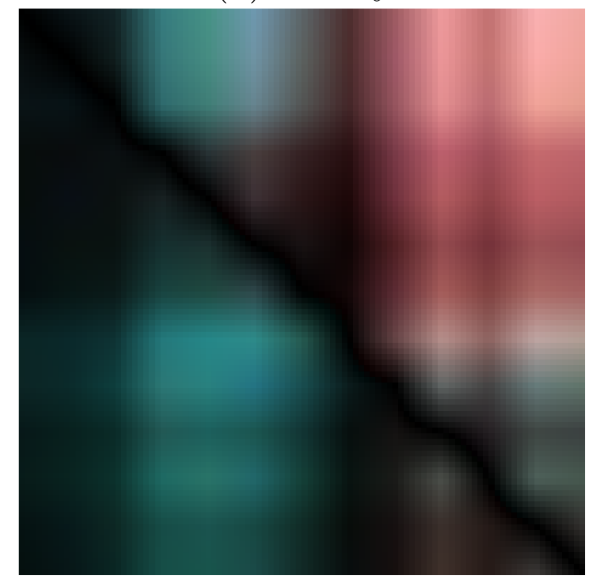

(b) Canola

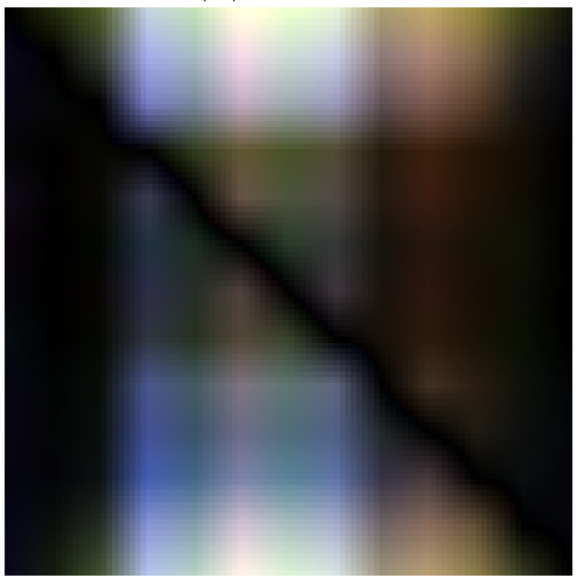

(c) Pea

Figure 3. Change matrices for different crop types. The abnormal bright and dark rows and columns in (a), (b) and (c) are a consequence of the incidence angle. Figure $(\mathrm{d})$ presents a Heatmap of Frobenius distances between the change matrices of different crop types 


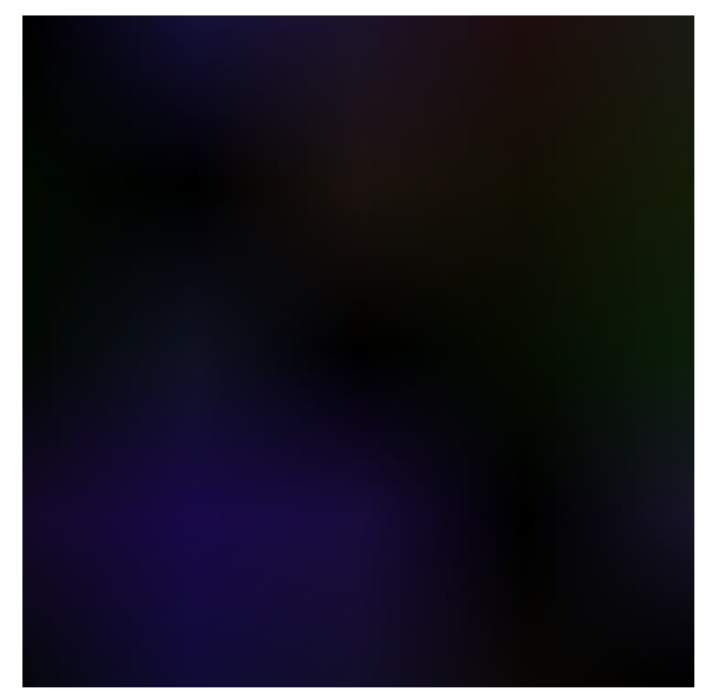

(a) River

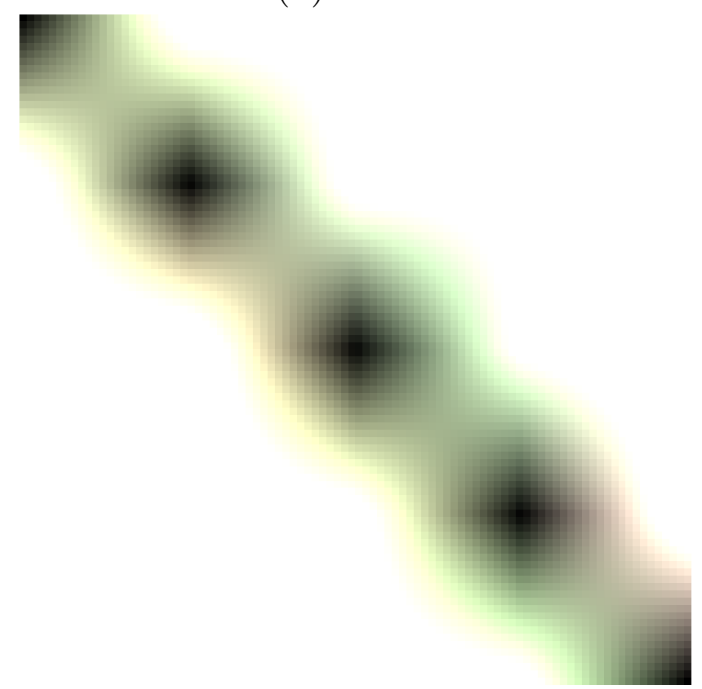

(c) City

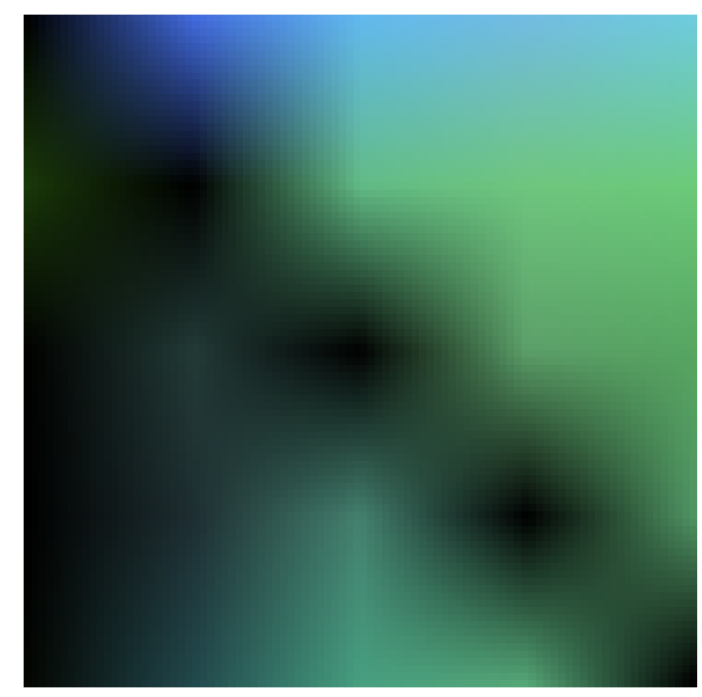

(b) Unknow crop 1

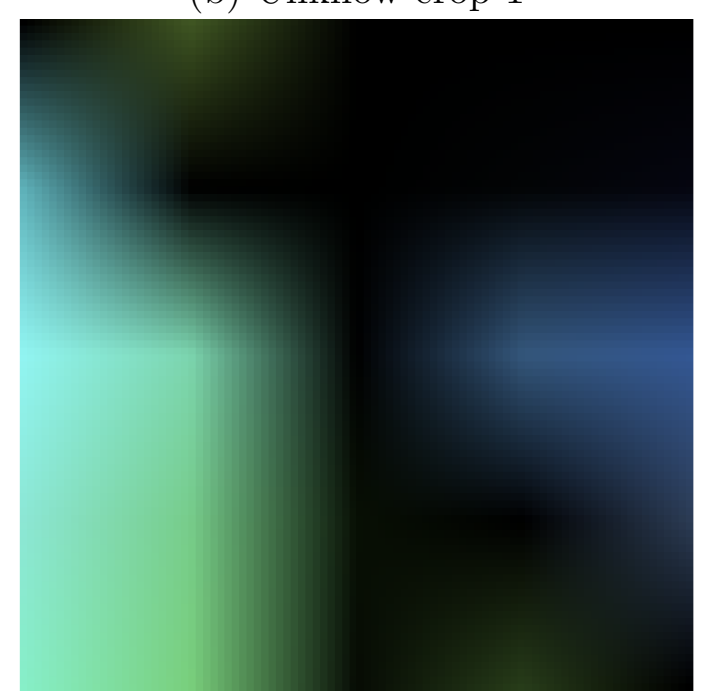

(d) Unknow crop 2

Figure 4. Change matrices for different land types in the Seville dataset. All change matrices in the document have the same color scaling for comparison purposes. 


\section{Land type classification based on the change matrix}

Every resolution cell in the image can be represented by the information contained in its change matrix. This encoded polarimetric multi-temporal information is compared with some model change matrices that represent the land classes in a supervised learning manner (training change matrices). A resolution cell is then labelled as belonging to the class to which it is more similar. To compare the similarity between a pixel change matrix and a class change matrix, the Euclidean distance between the two matrices is used. The methodology was applied over the stack of images in the Seville test site (fig. 1). The results of part of the image are shown in figure 5 , considering initially 8 land type classes.

It can be seen that the most significant land types such as the river, the city and the rice fields are correctly identified. Further research is being carried out to better select the training change matrices and to determine the accuracy of the classification results.

\section{Acknowledgement}

This research was partially supported by the Project EO4cultivar, led by Environment Systems Ltd and co-funded by the UK Space Agency.

This work was additionally funded in part by the Spanish Ministry of Science and Innovation, the State Agency of Research (AEI) and the European Funds for Regional Development (EFRD) under Project TEC2017-85244-C2-1-P.

RADARSAT-2 Data and Products (C) MacDonald, Dettwiler and Associates Ltd. (2014) - All Rights Reserved. Radarsat-2 is an official trademark of the Canadian Space Agency. The data over the rice fields were provided under the SOAR Education International Initiative (SOAR-EI-5158). The Agrisar images were provided by MDA and CSA in the framework of the ESA-funded AgriSAR2009 campaign. 


\section{Change matrix difference}

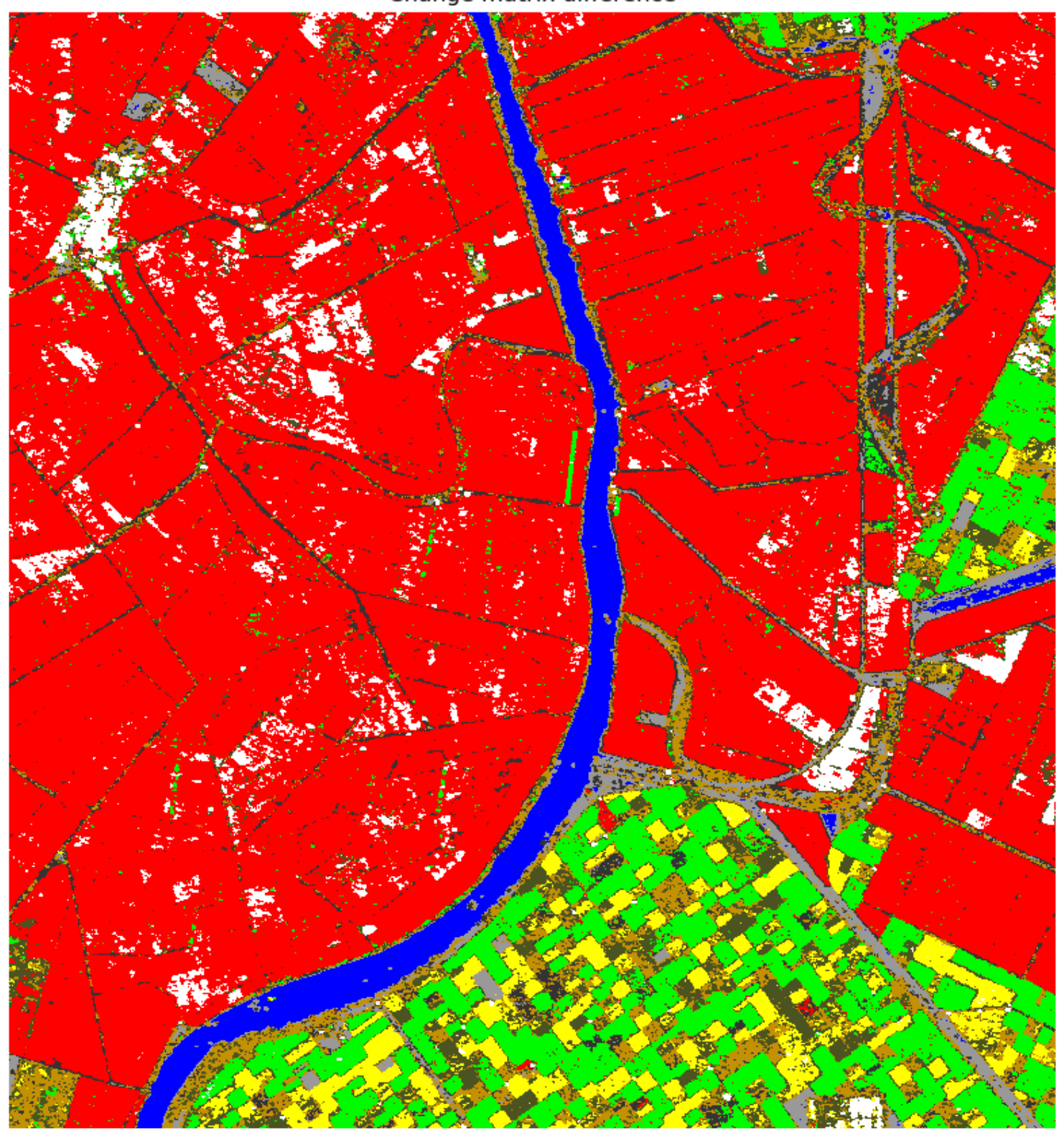

Figure 5. Multi-temporal classification based on the change matrix. The classes include the River (blue), rice fields (red), city (white), roads(black), unknown crop types(green and brown colours. The white patches in the rices fields correspond to roughness in the water caused by wind at beginning of the season. 


\section{REFERENCES}

[1] A. Alonso-González, T. Jagdhuber and I. Hajnsek, "Agricultural monitoring with polarimetric SAR time series," 2015 8th International Workshop on the Analysis of Multitemporal Remote Sensing Images (Multi-Temp), Annecy, 2015.

[2] A. Marino and A. Alonso-Gonzalez, "Optimisations for different change models with polarimetric SAR," EUSAR 2018; 12th European Conference on Synthetic Aperture Radar, Aachen, Germany, 2018, pp. 1-5.

[3] C. Silva, A. Marino, J. M. Lopez-Sanchez and I. Cameron, "Monitoring Agricultural Fields Using an Optimisation of the Difference of Covariance Matrices for Polsar," IGARSS 2018 - 2018 IEEE Igarss Symposium, Valencia, 2018.

\section{References}

